\title{
A COUNTEREXAMPLE TO A THEOREM OF BREMERMANN ON SHILOV BOUNDARIES
}

\author{
MAREK JARNICKI AND PETER PFLUG
}

(Communicated by Franc Forstneric)

\begin{abstract}
We give a counterexample to the following theorem of Bremermann on Shilov boundaries: if $D$ is a bounded domain in $\mathbb{C}^{n}$ having a univalent envelope of holomorphy, say $\widetilde{D}$, then the Shilov boundary of $D$ with respect to the algebra $\mathcal{A}(D)$, call it $\partial_{S} D$, coincides with the corresponding one for $\widetilde{D}$, called $\partial_{S} \widetilde{D}$.
\end{abstract}

Let us first repeat some basic notions: let $D$ be a bounded domain in $\mathbb{C}^{n}$. Put $\mathcal{A}(D):=\mathcal{C}(\bar{D}) \cap \mathcal{O}(D)$. Then $\mathcal{A}(D)$ together with the supremum norm $\|\cdot\|_{\bar{D}}$ is a Banach algebra of functions on $\bar{D}$. Moreover, we set $\mathcal{B}(D)$ as the closure of $\mathcal{O}(\bar{D})$ in $\mathcal{C}(\bar{D})$ with the above norm. Again this is a Banach algebra. Then both of these algebras have a Shilov boundary, called $\partial_{S} D$, respectively, $\partial_{B} D$. Obviously, we have $\partial_{B} D \subset \partial_{S} D$.

Now we assume in addition that $D$ has a univalent envelope of holomorphy $\widetilde{D}$. Then, we have

$$
\partial_{S} \widetilde{D} \subset \partial_{S} D \subset \partial D \text { and } \partial_{B} \widetilde{D} \subset \partial_{B} D \subset \partial D .
$$

In Bre 1959] (see Theorem in section 6.6), Bremermann claimed that equality $\partial_{S} \tilde{\widetilde{D}}=\partial_{S} D$ is also true. While he proved $\partial_{S} \widetilde{D} \subset \partial_{S} D$ in a detailed way he was saying that the inverse inclusion is an obvious fact. But as we will show (more than fifty years later) this claim is not true, already if $D$ is a Hartogs domain over an annulus. For positive results regarding Reinhardt domains see [Kos-Zwo 2013].

Following the construction of some Hartogs domain with non univalent envelope of holomorphy (see Jar-Pfl 2000, pages 1-2) we get the following result.

Theorem. There exists a bounded Hartogs domain $D \subset \mathbb{C}^{2}$ with a univalent envelope of holomorphy $\widetilde{D}$ such that

- $\partial_{S} D \neq \partial_{S} \widetilde{D}$,

- $\partial_{B} D \neq \partial_{B} \widetilde{D}$,

- there exists a function $f \in \mathcal{O}(\bar{D})$ such that the holomorphic extension $\tilde{f}$ of $\left.f\right|_{D}$ to $\widetilde{D}$ has no continuous extension to $\widetilde{\widetilde{D}}$.

Received by the editors September 14, 2013.

2010 Mathematics Subject Classification. Primary 32D10, 32D15, 32D25.

Key words and phrases. Shilov boundary, Bergman boundary.

The research was partially supported by grant no. UMO-2011/03/B/ST1/04758 of the Polish National Science Center (NCN). 
Proof. Let $A:=\{z \in \mathbb{C}: 1 / 2<|z|<1\}$. Then we introduce

$$
\begin{aligned}
D: & =\left\{z \in A \times \mathbb{C}: \operatorname{Re} z_{1}<0,\left|z_{2}\right|<3\right\} \\
& \cup\left\{z \in A \times \mathbb{C}: 0 \leq \operatorname{Re} z_{1}, \operatorname{Im} z_{1}>0,\left|z_{2}\right|<1\right\} \\
& \cup\left\{z \in A \times \mathbb{C}: 0 \leq \operatorname{Re} z_{1}, \operatorname{Im} z_{1}<0,2<\left|z_{2}\right|<3\right\} .
\end{aligned}
$$

Note that $D$ is a Hartogs domain over the annulus $A$ which cuts the base $A \times\{0\}$. Then Corollary 3.1.10(b) in Jar-Pfl 2000 implies that $D$ has a univalent envelope of holomorphy $\widetilde{D}$. Moreover, using the Cauchy integral formula shows that $\widetilde{D}$ contains the following domain

$$
\left.\left\{z \in A \times \mathbb{C}: \operatorname{Re} z_{1}<0 \text { or (if } \operatorname{Re} z_{1} \geq 0 \text {, then } \operatorname{Im} z_{1}<0\right),\left|z_{2}\right|<3\right\} .
$$

In particular, all the discs $\mathbb{D}_{z_{1}}:=\left\{z_{1}\right\} \times 3 \mathbb{D}, 1 / 2 \leq \operatorname{Re} z_{1}=z_{1} \leq 1$, belong to $\overline{\widetilde{D}}$ (here $\mathbb{D}$ means the open unit disc in $\mathbb{C}$ ).

Therefore, if $f \in \mathcal{A}(\widetilde{D})$, then $f\left(z_{1}, \cdot\right) \in \mathcal{A}(3 \mathbb{D})$ for all the above $z_{1}$ (use Weierstrass' theorem here). Hence, by the maximum principle, these discs don't contain any point of $\partial_{S} \widetilde{D}$.

On the other side we discuss the following domain

$$
\begin{aligned}
D^{\prime}: & =\left\{z \in A^{\prime} \times \mathbb{C}: \operatorname{Re} z_{1}<\varepsilon\left|\operatorname{Im} z_{1}\right|,\left|z_{2}\right|<3+\varepsilon\right\} \\
& \cup\left\{z \in A^{\prime} \times \mathbb{C}: 0 \leq \operatorname{Re} z_{1}, \operatorname{Im} z_{1}>-\varepsilon \operatorname{Re} z_{1},\left|z_{2}\right|<1+\varepsilon\right\} \\
& \cup\left\{z \in A^{\prime} \times \mathbb{C}: 0 \leq \operatorname{Re} z_{1}, \operatorname{Im} z_{1}<\varepsilon \operatorname{Re} z_{1}, 2-\varepsilon<\left|z_{2}\right|<3+\varepsilon\right\},
\end{aligned}
$$

where $0<\varepsilon \ll 1 / 4$ and $A^{\prime}:=\{z \in \mathbb{C}: 1 / 2-\varepsilon<|z|<1+\varepsilon\}$. Observe that $D \subset \subset D^{\prime}$.

Now we define the following concrete holomorphic function $g$ on $D^{\prime}$ :

$$
g(z):=\left\{\begin{array}{ll}
\log _{1} z_{1}, & \text { if } z \in D^{\prime},\left|z_{2}\right|>1.4 \\
\log _{2} z_{1}, & \text { if } z \in D^{\prime},\left|z_{2}\right|<1.6
\end{array},\right.
$$

where $\log _{1}$, respectively $\log _{2}$, is the branch of the logarithm function on $\mathbb{C} \backslash\{w \in \mathbb{C}$ : $\operatorname{Re} w \geq 0, \operatorname{Im} w=\operatorname{Re} w\}$, respectively on $\mathbb{C} \backslash\{w \in \mathbb{C}: \operatorname{Re} w \geq 0, \operatorname{Im} w=-\operatorname{Re} w\}$, with $\log _{1}(-1 / 2)=\log _{2}(-1 / 2)=\log 1 / 2+i \pi$. Observe that $g$ is well defined on $D^{\prime}$ and $g \in \mathcal{O}\left(D^{\prime}\right)$.

Define $f:=\left.g\right|_{\bar{D}}$. Then $f \in \mathcal{B}(D)$ and if $z \in \bar{D}$ with $z_{1}=\operatorname{Re} z_{1}>0$, then

$$
f(z)=\left\{\begin{array}{ll}
\log \operatorname{Re} z_{1}, & \text { if }\left|z_{2}\right| \leq 1 \\
\log \operatorname{Re} z_{1}+2 \pi i, & \text { if }\left|z_{2}\right| \geq 2
\end{array} .\right.
$$

Finally, we observe that the function $h$ defined as $h(z):=\exp (i f(z)+2 \pi), z \in \bar{D}$, belongs to $\mathcal{B}(D)$ and

$$
|h(z)|= \begin{cases}1, & \text { if } z \in \bar{D}, \operatorname{Re} z_{1}>0,\left|z_{2}\right| \geq 2 \\ e^{2 \pi}, & \text { if } z \in \bar{D}, \operatorname{Re} z_{1}>0,\left|z_{2}\right| \leq 1\end{cases}
$$

and $|h(z)|<e^{2 \pi}$ on the remaining part of $\bar{D}$. Therefore, $\partial_{B} D$ contains points $z \in \bar{D}$ with $0<\operatorname{Re} z_{1}=z_{1}$ and $\left|z_{2}\right| \leq 1$.

Combining this concrete information with the general one from the former discussion on the Shilov boundaries for $\widetilde{D}$ we conclude that $\partial_{S} \widetilde{D}$ and $\partial_{B} \widetilde{D}$ are strictly contained in $\partial_{B} D$. In particular, this shows that the claimed equality in Bremermann's paper does not hold. 
Moreover, the function $f$ is the one whose existence was claimed in the third claim in the theorem.

Remark. (a) Recall that the equality $\partial_{S} D=\partial_{S} \widetilde{D}$ is true for a Reinhardt domain D.

(b) Let $D$ be a bounded balanced domain. Obviously, $\bar{D}$ has a neighborhood basis of balanced domains $G$. Moreover, $D$ and each $G$ have univalent envelopes of holomorphy with $\widetilde{D} \subset \subset \widetilde{G}$. Hence the equality $\partial_{B} D=\partial_{B} \widetilde{D}$ holds in an obvious way.

(c) What remains is to discuss the equality of the Shilov boundaries for $\mathcal{A}(D)$ and $\mathcal{A}(\widetilde{D})$ in the case when $D$ is a balanced domain.

\section{REFERENCES}

[Bre 1959] H. J. Bremermann, On a generalized Dirichlet problem for plurisubharmonic functions and pseudo-convex domains. Characterization of Šilov boundaries, Trans. Amer. Math. Soc. 91 (1959), 246-276. MR0136766 (25 \#227)

[Kos-Zwo 2013] Łukasz Kosiński and Włodzimierz Zwonek, Proper holomorphic mappings vs. peak points and Shilov boundary, Ann. Polon. Math. 107 (2013), no. 1, 97-108, DOI 10.4064/ap107-1-7. MR3001625

[Jar-Pfl 2000] Marek Jarnicki and Peter Pflug, Extension of holomorphic functions, de Gruyter Expositions in Mathematics, vol. 34, Walter de Gruyter \& Co., Berlin, 2000. MR:1797263(2001k:32017)

Jagiellonian University, Faculty of Mathematics and Computer Science, Institute of Mathematics, Łojasiewicza 6, 30-348 Kraków, Poland

E-mail address: Marek.Jarnicki@im.uj.edu.pl

Carl von Ossietzky Universität Oldenburg, Institut für Mathematik, Postfach 2503 , D-26111 Oldenburg, Germany

E-mail address: Peter.Pflug@uni-oldenburg.de 